\title{
Extending the Service Life Span of Ageing Oil and Gas Offshore Production Facilities
}

\author{
Sushil Palkar $^{1}$ and Tore Markeset ${ }^{2}$ \\ ${ }^{1}$ Aker Solutions, Bergen, Norway \\ sushil.palkar@gmail.com \\ ${ }^{2}$ University of Stavanger, N-4036 Stavanger, Norway \\ tore.markeset@uis.no
}

\begin{abstract}
A large number of facilities and parts of the infrastructure of offshore oil and gas reservoirs worldwide are approaching or have exceeded their original design life. The petroleum fields are still producing substantial levels of hydrocarbons which are recoverable and profitable if the field's lifetime is extended. Thus, parts of this infrastructure are being considered for use beyond their planned design life. However, focusing on safety considerations, the condition of systems, structures and components (SSC) may not be acceptable for extended operation. The purpose of this paper is to discuss guidelines for assessing life extension in order to ensure the technical and operational integrity of these ageing facilities. The objective is to highlight those mechanisms by which the installation is degraded physically and functionally including the human factors and organizational issues. This documentation on ageing mechanisms will provide the foundation for a service lifetime extension process.
\end{abstract}

Keywords: Ageing oil and gas production facilities, Maintenance, Extension of petroleum production facility service life span.

\section{$1 \quad$ Introduction}

\subsection{Background}

Many offshore oil \& gas installations are in the life extension stage, as they have passed their original design life. There will be a time when an installed facility has to be closed down permanently as per the original design life. However, with certain processes and criteria, the life of this facility can be extended without compromising the safe working limits. This concept of increasing the life of a facility without increasing the risk is termed as Lifetime Extension [1].

\subsection{Problems Description}

There are many factors that influence the decision to extend the operation of a producing facility. Some of these include:

- The oil reservoir produces more oil than estimated.

- The advancement in techniques related to extraction of oil allows greater extraction. 
- Improved techniques to access seismic data provide more details about the reservoirs.

- The provision of processing capability at a nearby subsea or minimum facility platform.

When planning for life extensions of old platforms, it is beneficial to review the integrity management systems and the manpower required to manage an ageing installation. There are a number of threats to integrity from physical degradation mechanisms that are time-dependent and therefore increase with the age of an installation.

\subsection{Main Objective}

The main objective of this paper is to provide some guidelines for assessing life extension in order to ensure the technical and operational integrity of these ageing facilities. Furthermore, the paper will identify aspects of ageing management (material degradation, obsolescence, human \& organizational concerns/issues), the main tasks of the life extension process, and some of the knowledge gaps. Suggestions and recommendations will be made for the lifetime extension process.

\subsection{Research Method}

The report is based on a broad literature study together with discussions and interviews with oil and gas industry specialists. Industry standards and guidelines are used to develop the report.

\section{Identify Aspects of Ageing Management}

The management of ageing offshore facilities is an important issue for companies that intend to extend the lifetime. To extend the life span of offshore facilities, installations must be sufficiently safe and economical to remain in operation. This can be achieved by identifying aspects of the management of ageing and implementing them to control and mitigate the degradation of the facilities. Material degradation, obsolescence, and organizational issues are the three aspects of ageing. These will be discussed in the following.

\subsection{Material Degradation}

This is a broad term encompassing some of the major reasons for ageing and therefore the reason for the life extension process. The degradation can be associated with physical, operational and environmental factors. Also, major unfavorable maintenance practices can lead to material degradation.

- Corrosion - Loss of material due to electro-chemical reaction with the environment.

- Internal - Most of the internal corrosion problem are due to the corrosive contents of the produced well fluids, such as dissolved gases e.g. $-\mathrm{CO}_{2}$ and $\mathrm{H}_{2} \mathrm{~S}$. The constitution of the well fluids changes with time and old fields tend to be sourer, leading to an increasing rate of corrosion.

- External - This can be due to offshore environments, with sea water in the air. Corrosion under cladding or coating (e.g. PFP, insulation) is a significant issue 
and difficult to detect. Corrosion of exposed steel work is an increasing problem of ageing installations, particularly if maintenance (repainting) is poor.

- Ageing of topside equipment includes the whole range of equipment placed on the topsides of any installations include wearing out of moving or rotating equipment due to friction, and erosion due to the removal of material from the fluid flow. This effect of degradation includes:

- Wearing out of moving or rotating equipment due to friction particularly.

- Erosion due to removal of material from fluid flow, particularly if the fluid contains solid particles, prior to separation on valve seats and other high velocity areas in the process.

- Environmentally assisted cracking: This is cracking due to electrochemical reaction of material with the environment, which includes stress corrosion cracking (SCC) and hydrogen embrittlement. The extent and rate of these processes are age related.

- General fatigue failures of welds and material: The structure is the primary mechanism of any installation whether fixed or floating, above or below water, which provides a supporting framework within a band of tolerance of movements that ensures the equipment and personnel continue to function properly \& safely. Structures are subjected to changing loads and susceptibilities (e.g. - Increasing loads due to the marine growth) causing fatigue (it is caused due to the development of cracks under cyclical stresses). There will be general fatigue due to failure of welds and materials due to repeated cyclic stresses and vibration fatigue caused due to high cycle low amplitude cyclic stresses due to poor fixing, resonance such as in small bore piping attachments.

- Accumulated damage: There may be substantial accumulated damages such as dents and gouges primarily due to the impact from objects dropped from the platform or attendant vessels or as a result of maintenance.

- Scour: Scour is the erosion of loose seabed material directly around offshore structures. This can increase the height of the structure subjected to hydrodynamic loading.

- Increased structural load: Changes in effective water depth can increase both hydrodynamic loading on the structure and the probability of the deck being inundated during extreme weather conditions; effective water depth can be increase due to - settlement, subsidence, vertical movements of the tectonic plates.

- Blockages: Blockages of pipe work, valves, heat exchanger tubes, pressure relief systems, etc., due to build-up of corrosion products, fouling and scaling, etc.

\subsection{Obsolescence}

Due to prolonged use, the equipment gradually becomes out-of-date and this signifies ageing. Equipment becomes out of date or back logged and this represents a form of ageing. For example - the corrosion management system may no longer be suitable for current product chemistry, backlogs can develop in planned maintenance \& inspection and the plans themselves may need to review or revise to reflect the state ageing equipment. In addition to outdated technology, obsolescence includes new needs, where one need gives rise to another. For example, to extract oil from reservoirs located further away from the facility and existing wells, new tie-ins and new types of wells are needed. This in turn results in a need for a new technology. An assessment of the extent and accuracy of available knowledge, and the adequacy of that knowledge to make sound judgments, is an essential part of the life extension process. 


\subsection{Organizational Issues}

Usually both the work force and the installation platform are ageing simultaneously and therefore the transfer of information may not function properly. The workforce, the team dedicated or allotted for particular installation, age and change, therefore level of knowledge and preparedness particularly in the event of emergency or crisis, have to be regularly tested and refreshed especially for their influence on or participation in any proposed management system. Arrangements for maintaining a trained and competent workforce with an awareness of equipment ageing and its effects is an issue to be addressed. Much of the current workforce is acknowledged to be approaching retirement and the succession needs to be part of life extension planning. Loss of corporate knowledge with retiring staff is also an issue.

The teams that are ageing are more than just operational teams or design teams that support as well as undertake new builds. On account of very limited new people entering in teams, succession of knowledge is hindered. Also the teams represent a significant proportion of organisational memory. Usually both the work force and the installation platform are ageing simultaneously and therefore the transfer of information mechanism may not function properly.

Any degradation or problem on the installation if not managed in a good way, may pass on a message of corporate negligence to the staff working offshore which will in some way affect their acceptance of any new integrity management scheme. Thus extension planning can help to ensure that the organizational issues and knowledge are up to date and hence they are not hampering any progress. Also the success of management scheme developed to manage life extension should be in accordance with abilities, skills and aptitude of the workforce who are intended to implement them.

\section{Identify the Main Tasks of Life Extension}

To maintain the structural and functional integrity of the equipment in order to carry out life extension, good knowledge of past and present condition of the equipment is of utmost importance. The main task is to check whether the equipment is functional and fit for purpose. It is suggested that the lifetime extension process should include the following activities, which will be discussed thereafter:

1. Strategic collection of data and information

2. System breakdown, criticality screening, monitoring and testing

3. Secondary screening and detailed analysis

4. Risk factors and assessment with respect to ageing

5. Screening of factors regarding obsolescence and organizational challenges

6. Possible challenges in the lifetime extension process with respect to degradation

7. Outline of the challenges identified

8. Estimation and evaluation of risk

9. Lifetime extension management plan

10. Uncertainties related to the lifetime extension 


\subsection{Strategic Collection of Data and Information}

This is the basic and most important step of the lifetime extension process. Various data and information from the initial phase to the time of installation and its working life should be collected and accessed to analyze the risk-producing and -reducing factors, including the (risk) acceptance criteria, without compromising on safety.

\section{The Design of the Equipment and Its Operating Life}

Typically 20-25 years from the time of its construction have been considered as the safe working life of an installation. The estimation of the design life of equipment is a useful measure to maintain the integrity of SSC (structure, system and components). Design life represents the limit of foresight and experience of the original designers. Moreover, it is necessary that we collect data about the design, fabrication and installation (DFI) which provides information about the different phases of installation [2].

During the process when more data is collected, gathered and shared, the following issues are highlighted:

- The local conditions and environment stresses

- What the installation demands in order to give satisfactory performance

- The organizational practices and safety regulations

- The type of work force working offshore, their cultures and attitudes.

\section{Important Checkpoints to Be Addressed}

There may be many checkpoints to deal with, of which a few basic points are:

- To check whether the equipment still satisfies the functional requirements and safety limits of its original specification, design and construction standards.

- To check whether equipment meets the latest standards. To check these, standard norms have been incorporated or applied on equipment.

- To check the quality of original fabrication.

- To check whether the equipment meets its current functional requirements, so that it is suitable for the purpose.

An assessment for good fabrication standards should be made, identifying the pros and cons. Signs of poor fabrication can be:

- Misalignment welds, partial penetration, weld repairs, welding spatter and defects.

- Poor finishing such as incomplete or thin paintings or coatings.

- Poor fitting joints, or overloaded seals, glands and gaskets, leak weeps.

- Vibrating and out-of-balance rotating equipment.

- Stiffness or looseness in moving parts and mechanisms.

- Insufficient fixtures and supports.

- Damage or excessive force applied during installation. 


\subsection{System Breakdown, Criticality Screening, Monitoring and Testing}

The screening activity is based on the classification of critical failures and the consequences of the failures of structures, systems and components (SSC). This is a riskbased approach inspired by NORSOK Z-008 [3]. Risk depends on both probability of failure $(\mathrm{PoF})$ and the consequence of failure $(\mathrm{CoF})$. The consequences can be categorized as 'high', 'medium' or 'low'. If the consequence for a function/system or SSC falls within the category 'high', further analysis (reliability centered maintenance actions) is required or the system needs to be redesigned or modified to reduce the risk with respect to cost and production loss, without compromising on safety. If the consequence falls in the category 'medium', it is not mandatory to carry out a detailed analysis (focusing on risk of major hazards); it should be sufficient to follow safety management/maintenance management systems. If the consequence falls in the category 'low', it is generally recommended to carry out planned corrective maintenance activities or to follow the maintenance activities recommended by the original equipment manufacturer [4].

\subsection{Secondary Screening and Detailed Analysis}

This criticality screening is based on the primary investigation for material degradation, obsolescence, and organizational issues. Now, for those SSC that have a high risk factor based on the primary screening, there should be a secondary screening, based on the aspects of ageing and acceptability for the lifetime extension process. This should be especially done for those SSC which are inaccessible for continuous and detailed inspection. In order to assess their state, they should be analyzed in detail with respect to material degradation. The critical SSC can be classified according to:

- Their availability for monitoring/inspection.

- Their accessibility for modification and maintenance.

\subsection{Risk Factors and Assessment with Respect to Ageing}

In reviewing the equipment for life extension, the effect of various risk-reducing measures should be analyzed - increased testing, replacement, etc. The workforce needs to be aware of any factors that could increase the risk and rate of ageing and their effects during the life of the equipment.

\subsection{Screening of Obsolescence and Organizational Challenges}

The following are the challenges, gaps and measures related to obsolescence and organizational issues [5]:

- Requirements - are they satisfactory?

- New operational conditions need to be anticipated for the lifetime extension period.

- Equipment being or becoming 'out-of-date', possibly causing challenges, e.g. related to availability of spare parts.

- Introduction of new technology foreseen for the lifetime extension period. 
- Possible reorganizations (e.g. introduction of integrated operations ${ }^{1}$ [6] or company merging).

- Maintaining personnel competence (e.g. ageing of personnel).

- Transfer of knowledge during the lifetime extension period.

It should be kept in mind that these two factors, i.e. new technology - new competence of maintenance operators, are closely intertwined.

\subsection{Possible Challenges in the Lifetime Extension Process with Respect to Material Degradation}

The possible challenges identified with respect to material degradation are as follows:

- Understanding the ageing mechanisms, failure causes, and modes of failure of the SSC. This is not possible unless and until a complete set of quality data is available.

- Understanding the good models to describe the degradation in terms of a specific parameter to have a clear picture of the damage.

- The choice of correct parameters to describe the current state of damage is also an important issue.

- Having sufficient knowledge and models for the effect of maintenance on the degradation process to be understood.

- Having sufficient competence and knowledge among the workforce to carry out the lifetime extension plan.

These challenges are only relevant depending upon the actual systems and how accessible they are in their current state of degradation.

\subsection{Outline of the Challenges Identified}

This section deals with the level of risk of the facility, within the acceptable limits during the execution process. Also, systems and components are checked and followed up for updating and maintenance in terms of risk related to major hazards (e.g. fire/explosion, dropped objects, structure collapse).

\subsection{Estimation and Evaluation of Risk}

Many system failures are consequential and not the result of one break or malfunction but of a sequence of many failures. As a result, reliability and risk analysis must not be conceived as a static exercise. Risk and reliability analysis is a dynamic process which must be updated and upgraded as additional information is available. In other words, the estimation of risk may be quite uncertain; this can be due to the difficulty of predicting the future state of some equipment [7]. If the probability of failure (PoF) and/or consequence of failure $(\mathrm{CoF})$ are considered to be very uncertain, one should not use 'best estimate' of these, but rather apply more conservative values. Usually those with uncertain estimated values result in higher risk.

\footnotetext{
${ }^{1}$ Integrated Operations is about ensuring people, new work processes and technology work much more efficiently together, in order to reach safe and better decisions faster.
} 


\subsection{Lifetime Extension Management Plan}

After risk assessment is carried out and resulting measures are implemented, a management plan for the lifetime extension should be prepared. Now we have a final 'action plan' consisting of risk-reducing measures (maintenance, modifications, other compensating measures and defenses) that should be implemented before the lifetime extension assessment plan and during the lifetime extension period.

\subsection{Uncertainties Related to the Lifetime Extension}

There are two types of uncertainties that exist related to probabilities of failure. They are Type (I) \& Type (II):

- Type (I) - Referred to as natural, physical, inherent, this incorporates natural randomness.

- Type (II) - Referred to as unnatural, modeling, cognitive, this incorporates the imprecise nature of theoretical models and our knowledge. These types of uncertainties are seen in the lifetime extension process.

Analysis capabilities may have improved, but often there is insufficient data to support the analyses and thus to predict a future state of an SSC.

\section{Conclusion and Suggestions}

The life extension process should be carried out for future economic consideration without compromising on safety standards. Life extension of fixed offshore installations can actually help to maintain their integrity (for example topsides equipment, pipelines, flexible risers, etc.), and therefore they can perform adequately giving good results.

It is always said that "Prevention is better than cure". Therefore periodic inspections of SSC can help operators to be informed about the current status of the installation. These inspections should be based on proper documentation with clear ideas and understanding so that dissemination of the knowledge is feasible. For example, information should be stored in the form of response measurement. Also response data can be used to calibrate and improve the design process of future systems.

An informed team/workforce is the first step toward the success of the lifetime extension assessment. Proper communication within the various departments such as process, piping, maintenance, mechanical, electrical, instrument, structural, project \& planning, safety, etc. is very important. Also, it is equally important to learn lessons during the operation which can be implemented in the next project. It is also important to share the knowledge because information and knowledge remain in a system and not just within an individual. This guarantees a more secure handover or changeover in staff.

The software system also plays an important role. It helps in intelligently analyzing the data, assessing the changes in integrity status and visually reporting back to the end user both strategic and detailed information. This is beneficial to help manage some of the problems associated with data management and data sharing. 


\section{References}

1. Palkar, S.: Lifetime Extension of Ageing Oil and Gas Platforms. Master Thesis University of Stavanger, Stavanger, Norway (June 2010)

2. Wintle, J., Sharp, J.: TWI REPORT 17554/1/08 - Requirements for Life Extension of Ageing Offshore Production Installations. For Petroleum Safety Authority (2008)

3. NORSOK STANDARD - Z-008: Criticality Analysis for maintenance purposes. Rev. 2 (2001)

4. Panesar, S.S., Kumar, R., Markeset, T.: Development of Maintenance Strategies for Offshore Production Facilities. In: The Proceedings of the 3rd World Congress on Engineering Asset Management and Intelligent Maintenance Systems (WCEAM-IMS 2008), October 28-30, pp. 1227-1232. Beijing International Convention Center, Beijing (2008) ISBN 978-1-84882-216-0

5. Hokstad, P., Håbrekke, S., Johnsen, R., Sangesland, S.: Ageing and life extension for offshore facilities in general and for specific systems. SINTEF Report for the Petroleum Safety Authority Norway (2010)

6. Jansen, B., Høydalsvik, H., Nordtvedt, J.E., Håvard, M.: Potential Value of Integrated Operations on the Norwegian Shelf. Study by Epsis and ABB commissioned by OLF Norwegian Oil Industry Association (2006)

7. Kumar, U.: Risk based maintenance strategies for mechanised and automated systems. University of Stavanger, Norway 